\title{
Doxorubicin Supplemented with Pravastatin in Lipid Nanoemulsion Induces Antineoplastic Activity with Limited Hepatotoxicity and Cardiotoxicity in Tumor-Bearing Mice
}

\author{
Mayson H. Alkhatib ${ }^{1,2^{*}}$, Huda M. Alkreathy ${ }^{3}$, Mashael I. Al Omar', Khadijah S. Balamash', \\ Faiza Abdu ${ }^{4}$ and Ahmad Esmat ${ }^{3,5}$ \\ 'Department of Biochemistry, Faculty of Science, King Abdulaziz University, Jeddah, Saudi Arabia; \\ mhalkhatib@kau.edu.sa \\ ${ }^{2}$ Regenerative Medicine Unit King Fahd Center for Medical Research, King Abdulaziz University, Jeddah, Saudi \\ Arabia \\ ${ }^{3}$ Department of Pharmacology, Faculty of Medicine, King Abdulaziz University, Jeddah, Saudi Arabia \\ ${ }^{4}$ Department of Biological Sciences, Faculty of Science, King Abdulaziz University, Jeddah, Saudi Arabia \\ ${ }^{5}$ Department of Pharmacology and Toxicology, Faculty of Pharmacy, Ain Shams University, Cairo - 11566, Egypt
}

\begin{abstract}
Doxorubicin (DXR) is widely indicated as anticancer drug, but serious cardiotoxicity limits its clinical application. Recently, Pravastatin (PS) is one of the statins that appear to possess a potential role in cancer therapy despite its hepatotoxicity. Interestingly, drug delivery systems are designed for targeted and controlled delivery of one or more drugs loaded in nanoparticles, holding an enormous potential in therapeutics. Therefore, the aim of the current study was to assess the tolerability of a novel nanoemulsion formulation holding DXR and pravastatin (DXR+PS/LNE) in Swiss albino mice bearing Ehrlich Ascites Carcinoma (EAC). The efficacy and tolerability of nanoemulsion formulation was assessed by monitoring body weight changes, biochemical and histopathological profiles of cardiac and hepatic tissues. The formulated DXR+PS/ LNE has mean droplet diameter of $139.90 \pm 3.85 \mathrm{~nm}$. The present findings indicated that DXR+PS/LNE caused a significant decrease in body weight change and a $217.35 \%$ increase in the mean survival time compared to EAC-challenged mice. In addition, no significant changes in biochemical parameters were detected compared to corresponding controls. The current preclinical results suggest that the nanoemulsion formulation of doxorubicin with pravastatin could be a promising novel cancer therapy, in terms of tolerability.
\end{abstract}

Keywords: Cardiac Tissue, Ehrlich Ascites Carcinoma, Hepatic Tissue, Mean Survival Time, Statins, Transmission Electron Microscope

\section{Introduction}

Cancer is a potentially fatal ailment, caused by uncontrolled proliferation and metastases of abnormal cells. Chemotherapy can be defined as the use of anticancer drugs either single or in combination with other drugs to restrict the cancer progression. Various frontline chemotherapeutic agents, employed in oncology, are having many challenges like rapid drug clearance, low water solubility, low tolerability and non-specific tumor targeting; thereby damaging healthy cells during the treatment ${ }^{1}$.

${ }^{*}$ Author for correspondence 
Doxorubicin (DXR) is an effective and wide spectrum anticancer drug used to treat numerous cancer types, including breast carcinoma, hepatocellular carcinoma, ovarian carcinoma, and acute lymphoblastic leukemia. Nevertheless, its clinical application is limited due to its fatal cardiotoxic effects and dose-limiting myelosupression ${ }^{2}$. Many research studies proposed multiple approaches to reduce the effective dose of DXR and thereby its adverse effects ${ }^{3}$. DXR was incorporated into different drugs and natural products in different nanocarriers with the aim to improve its efficacy and eliminate its adverse side effects ${ }^{4-10}$.

Statins are a drug class used for treating hypercholesterolemia by selective inhibition of 3-hydroxy-3-methylglutaryl coenzyme A (HMG-CoA) reductase enzyme. They have pleiotropic effects like anti-inflammatory effects, antiproliferative and immune modulatory effects, plaque stability and prevention of platelet aggregation. Statins are indicated to prevent and treat many diseases including coronary artery diseases ${ }^{11,12}$. Enormous research-based data reported that statins including pravastatin (PS) mediates inhibitory functions in tumor growth by upregulating apolipoprotein A1 thereby potentiating antineoplastic activity including the cytostatic effect (G1/S phase arrest), proapoptotic activity such as glioma, leukemia cell lines, neuroblastoma, melanoma and antimetastatic properties ${ }^{13,14}$.

A considerable number of studies were performed to assess the antitumor potential of statins with or without anticancer drugs. However, it is the combination of statins with other chemotherapeutic agents that could significantly improve the cytotoxicity and tolerability greater than drug alone ${ }^{8,9,15,16}$. In early metastatic cancers, a combination of statins with a cytotoxic agent was more effective, yielding synergistic effect in experimental models and clinical settings ${ }^{13,17,18}$.

There are conflicting reports associating statin use and cancer outcomes like mortality and/or survival rates $^{19}$. However, statins can enhance the efficacy of the chemotherapeutic agents and attenuate chemoresistance ${ }^{16}$. Moreover, statins prolonged the survival rates of hepatocellular carcinoma patients and reduced risk to colorectal cancer and breast cancer ${ }^{20-22}$.

Nanotechnology is a cutting-edge technology helpful in designing and developing novel drug delivery systems, offering increased drug loading and better drug permeation leading to enhanced bioavailability of the anti-tumor drugs. Nanoemulsion (NE) is a colloidal heterogeneous system comprising of two immiscible liquids in which liquid droplets of one liquid (with diameters ranging from 1 to $100 \mathrm{~nm}$ ) are dispersed in the other liquid ${ }^{23}$. Anti-tumor drugs loaded in NE systems are more effective against cancer cells as compared to conventional delivery systems. This could be attributed to the synthesis of a thermodynamically stable aqueous dispersion system with lesser polydispersity index, smaller particle size and zeta potential by superior drug stability in the $\mathrm{NE}^{24,25}$.

Therefore, the aim of the current study was to assess the tolerability of a novel nanoemulsion formulation holding DXR and pravastatin (DXR+PS/LNE) in Swiss albino mice bearing Ehrlich Ascites Carcinoma (EAC).

\section{Materials and Methods}

\subsection{Materials}

DXR hydrochloride and PS sodium were procured from Pharmacopeia (CA, USA). 1-Octanol was obtained from Afla Aesar (GmbH \& Co KG Karlsruhe, Germany). Sodium oleate (SO), Soya phosphatidyl choline (SPC), polyoxyethylene glycerol trihydroxy stearate 40 (Eumulgin HRE 40, EU) and tris (hydroxymethyl) amino methane, were purchased from Leo Chem India (Seshadripuram, Bengaluru, India). Cholesterol as the oil phase was purchased from Jechno Pharmacies (Bahadurgarh, Haryana, India). Water purification system (Bibby Sterilin ltd, UK) was used to freshly prepare distilled water. Pravastatin (PS) was generously gifted by Jamjoom Pharma, Jeddah, Saudi Arabia.

\subsection{Experimental Animals}

Adult female mice (Swiss albino) of 9 weeks old weighing between $(25-30 \mathrm{~g})$ were used throughout this study. The mice were housed in big animal cages having maximum five mice per cage. They were kept under normal conditions $\left(\left(23^{\circ} \pm 2^{\circ} \mathrm{C} ; 12\right.\right.$ hour dark/light cycle) and were provided with standard pellet feed and drinking water ad libitum. Cells of Ehrlich ascites carcinoma (EAC) were purchased from American Type Tissue Culture Collection (Manassas, VA, USA). The current study was performed in accordance with the guidelines for the care and use of laboratory animals approved by the Institutional Animal Ethics Committee, Faculty of Medicine, King Abdulaziz University, Jeddah, Saudi Arabia (approval ref. no. 189/247/1433). 


\subsection{Methods}

\subsubsection{Nanoemulsion Preparation}

Preparation of NE formulation was carried out as described elsewhere ${ }^{25}$. Briefly, solid surfactants (1.25\% $\mathrm{w} / \mathrm{w})$ were mixed in a preset ratio of $3.5(\mathrm{EU}): 3.0(\mathrm{SPC})$ : 3.5 (SO). This was followed by the addition of cholesterol $(0.125 \% \mathrm{w} / \mathrm{w})$ as an oil phase gradually to the surfactant mixture pending the formation of a semisolid phase. Thereafter, 1 -octanol $(1 \% \mathrm{w} / \mathrm{w})$ was added drop by drop. At last, the aqueous phase containing warm Tris- $\mathrm{HCl}$ buffer ( $97.63 \% \mathrm{w} / \mathrm{w}$; $\mathrm{pH} 7.22)$ was added to dilute the mixture. A clear and transparent mixture was obtained by keeping the mixture in a water bath at $75^{\circ} \mathrm{C}$ for 3 hours which resulted in NE formulation. The resulting formulation was stored at $25^{\circ} \mathrm{C}$.

Various NE formulations used in the study were as follows: blank nanoemulsion (BL-NE), DXR loaded-LNE (DXR/LNE), prepared by dissolving DXR ( $2 \mathrm{mg} / \mathrm{kg}$ mouse body weight (BW)) in $0.2 \mathrm{ml}$ of LNE. Combined DXR-PS loaded-LNE at 1:2 ratio (DXR+PS/LNE), produced by dissolving PS (4 mg/kg mouse BW) in $0.2 \mathrm{ml} \mathrm{NE}$ with DXR ( $2 \mathrm{mg} / \mathrm{kg}$ mouse BW). PS loaded-LNE (PS/LNE), formed by solubilizing PS ( $4 \mathrm{mg} / \mathrm{kg}$ mouse BW) in $0.2 \mathrm{ml}$ NE. The $0.2 \mathrm{ml}$ of other treatment groups were prepared by solubilizing the drugs in $0.2 \mathrm{ml}$ of distilled water instead of $0.2 \mathrm{ml}$ of NE including DXR-Solution (DXRSol), and the Combination-Solution (DXR+PS-Sol).

\subsubsection{Transmission Electron Microscope (TEM)}

The morphology and droplet size distribution of the LNE formulations were viewed using TEM (CM 100, Philips, Holland). Briefly, a sample droplet was applied on copper grids of the electron microscope which were stained with phosphotungstic acid (PTA) $2 \%(\mathrm{w} / \mathrm{v})$ for $30 \mathrm{sec}$, and then excess acid was wiped with filter paper. It was followed by washing the grid with distilled water and then excess water was wiped with filter paper. After drying, the sample was observed using the TEM ${ }^{25}$.

\subsubsection{In-vivo anti-tumor activity}

The in-vivo antitumor activity was evaluated for various drug formulations as per the regimen suggested elsewhere ${ }^{26}$. Briefly, EAC cells were retained in-vivo in the ascetic form by repeated passages in the mice every $10^{\text {th }}$ day. On the $8^{\text {th }}$ day following transplantation, the ascetic fluid was withdrawn from EAC bearing mice. Prior to injection, viable EAC cells were collected from the peritoneal cavity and counted using the trypan blue exclusion test.

The mice were allocated into eight groups (20/group) as described in (Table 1). The body weight of each mouse was recorded prior to injecting with EAC cells. All groups were intraperitoneally injected with $\left(2 \times 10^{6}\right.$ cells/mouse $)$ on day 0 except group I kept without injection. Group I was the control while group II was considered as EACchallenged group. After 24 hours, animals in all remaining groups (III-VIII) received treatments every alternate day as illustrated in (Table 1).

Table 1. Treatment Allocation for Groups $(n=20)$

\begin{tabular}{|c|c|}
\hline Group & Treatment \\
\hline Group I & Normal - Saline $(200 \mu \mathrm{L})$ \\
\hline Group II & EAC challenged \\
\hline Group III & BL-NE $(0.2 \mathrm{~mL})$ \\
\hline Group IV & $\begin{array}{l}\text { DXR/LNE (2mg DXR/Kg) solubilized in } 200 \\
\mu \mathrm{L} N E\end{array}$ \\
\hline Group V & DXR-Sol (2 mg DXR/Kg) in $200 \mu \mathrm{L}$ saline \\
\hline Group VI & $\begin{array}{l}\text { DXR+PS/LNE (2 mg DXR/Kg with } 4 \mathrm{mg} \text { of } \\
\mathrm{PS} / \mathrm{Kg} \text { ) solubilized in } 200 \mu \mathrm{L} \mathrm{NE}\end{array}$ \\
\hline Group VII & $\begin{array}{l}\text { DXR+PS-Sol ( } 2 \text { mg DXR/Kg with } 4 \mathrm{mg} \text { PS/ } \\
\mathrm{Kg}) \text { in } 200 \mu \mathrm{L} \text { saline }\end{array}$ \\
\hline Group VIII & $\begin{array}{l}\text { PS/LNE (4 mg PS/Kg) solubilized in } 200 \mu \mathrm{L} \\
\mathrm{NE})\end{array}$ \\
\hline
\end{tabular}

On the $15^{\text {th }}$ day, 10 mice (from each group) were kept fasting for 24 hours, and BW of individual mouse was recorded. Blood samples were collected from retroorbital plexus for assessment of biochemical parameters. Then, animals were sacrificed by cervical dislocation. Then, the representative hepatic and cardiac tissues were resected from each group and kept in $10 \%$ formalin saline solution for histopathological examination. The remaining animals (ten per group) were set aside for the survival study.

\subsubsection{Organs Weight}

Instantaneously after sacrifice, the heart and liver from all animals were excised, cleansed with normal saline, dried against blotting paper and then weighed using calibrated weighing balance. The organ weight-to-body weight ratio was calculated as follows: 
Organ - to - bodyweight ratio $=\frac{\text { Post }- \text { sacrifice organs (heart or liver) weight }}{\text { Pre }- \text { sacrifice body weight of the animal }}$

\subsubsection{Biochemical Parameters}

A fully automated COBAS 8000 modular analyzer was used to assess the biochemical assays by following the standard kit methods. Briefly, blood samples were kept to clot and serum was separated by centrifugation at 5000 rpm for $10 \mathrm{~min}$. Sera were used to estimate biochemical hepatic parameters like alanine aminotransferase (ALT), aspartate aminotransferase (AST), alkaline phosphatase (ALP), and bilirubin (BIL). In addition, cardiac enzymes as creatine phosphokinase-MB (CK-MB) and lactate dehydrogenase (LDH) were also assessed.

\subsubsection{Histological Evaluation}

Paraffin blocks were prepared for hepatic and cardiac tissue, and then cut at 4 micron thickness by means of a microtome. After deparaffinization, slides were stained with hematoxylin and eosin $(\mathrm{H} \& \mathrm{E})$, then examined under light microscope (Eclipse 50i, Nikon Corporation, Japan).

\subsubsection{Survival Study}

The survival study started by observing the animals from the first week of experiment until the end of the study, i.e. $12^{\text {th }}$ week. Daily monitoring and recording of the mortality were done for 60 days to assess the mean survival time (MST), increased life span percentage (\% ILS) and survival percentage (\% S). The MST, \% ILS and $\% \mathrm{~S}$ were calculated based on the following equations ${ }^{27}$.

$$
\begin{aligned}
& \text { MST }=\frac{\text { (Day of the first death }+ \text { Day of the last death) }}{2} \\
& \% \mathrm{ILS}=\left[\frac{\text { (Mean survival time of treated group) }}{\text { (Mean survival time of control group) }}-1\right] \times 100 \\
& \% \mathrm{~S}=\frac{\text { (No. of animals surviving after treatment period) }}{\text { (No.of animals before treatment period) }} \times 100
\end{aligned}
$$

\subsection{Statistical Analysis}

All values were expressed as mean $\pm \mathrm{SD}$ obtained from each experimental group $(\mathrm{n}=10)$. Statistical analysis was carried out using one-way analysis of variance (ANOVA) test followed by Tukey's post-hoc test. All statistical analyses were performed using GraphPad Instat software (version 3, San Diego, CA, USA). All graphs were prepared using GraphPad Prism software (version 5.01, San Diego, CA, USA). The significant difference was considered when $P$-value $<0.05$.

\section{Results}

\subsection{Morphological Characterization of the Drug-Loaded Nanoemulsion}

The morphology and droplet sizes of the drugs-LNE formulations (DXR/LNE, PS/LNE, DXR+PS/LNE) were distinctly seen in the TEM images. As illustrated in (Figure 1), drugs-LNE formulations have semi-spherical droplets. The mean droplet size of DXR/LNE was $8.50 \pm 1.57 \mathrm{~nm}$ (range $6.90-10.08 \mathrm{~nm}$ ) while the size of the mean droplets of PS/LNE was significantly increased to $21.92 \pm 3.91 \mathrm{~nm}$ (range $17.98-25.80 \mathrm{~nm}$ ) (Table 2). $\mathrm{DXR}+\mathrm{PS} / \mathrm{LNE}$ has mean droplet sizes of $139.95 \pm 3.85 \mathrm{~nm}$ (range 136.13-143.81 nm) which was significantly bigger than the mean droplet sizes of the other single drug-LNE formulations (Table 2). The homogenous distribution of droplets without aggregation or adhesion was observed in all drugs-LNE formulations. The observed percentage of the coefficient of variation (\% CV) for droplet size was less than $20 \%$.
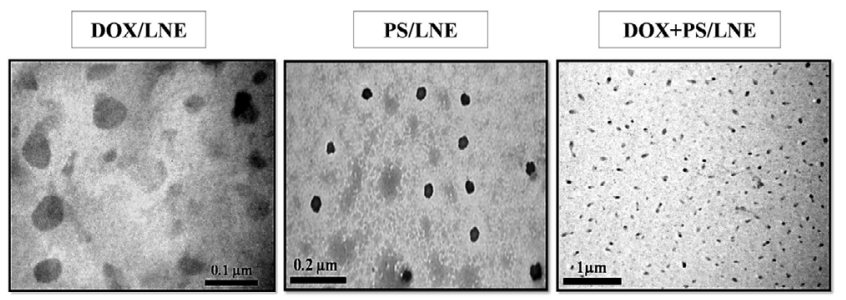

Figure 1. Microphotographs of DXR/LNE, PS/LNE, $\mathrm{DXR}+\mathrm{PS} / \mathrm{LNE}$ using transmission electron microscopy.

Table 2. TEM determined droplet size of the drugloaded nanoemulsion

\begin{tabular}{|c|c|c|c|}
\hline $\begin{array}{c}\text { Drug } \\
\text { Formulations }\end{array}$ & $\begin{array}{c}\text { Droplet } \\
\text { Diameter (nm) }\end{array}$ & $\begin{array}{c}\text { Droplet } \\
\text { Diameter } \\
\text { Range (nm) }\end{array}$ & \% CV \\
\hline DXR/LNE & $8.49 \pm 1.57 \#$ & $6.90-10.07$ & 18.56 \\
\hline PS/LNE & $21.92 \pm 3.90+$ & $17.97-25.80$ & 17.88 \\
\hline DXR+ PS/LNE & $139.90 \pm 3.85^{\star} \#$ & $136.13-143.81$ & 2.48 \\
\hline
\end{tabular}


Droplet diameters were expressed as mean $\pm \mathrm{SD}, \mathrm{n}=$ 10. \% CV: Coefficient of variation determined through dividing the standard deviation by the mean and then multiplying by 100 . Statistical analysis was carried out using one-way ANOVA followed by Tukey's post-hoc test. *Statistically significant differences from DXR/LNE at $\mathrm{p}<$ 0.05. "Statistically significant differences from PS/LNE at $\mathrm{p}<0.05$.

\subsection{Assessment of Toxic Effects of Different Formulations}

\subsubsection{Body Weight Change and Organ Weight Ratios}

As displayed in (Table 3), a significant body weight loss was observed in different formulations including DXR/ LNE, DXR-Sol, DXR+PS/LNE, DXR+PS-Sol and PS/ LNE groups in comparison with EAC-challenged group. Although, the average BW in the EAC-challenged group was higher than the control animals, a significant change in BW for BL-NE group was observed to be more than that change in EAC-challenged group. Interestingly, after 15 days the BW of both DXR soluble formulations (DXRSol and DXR+PS-Sol) treated groups were obviously less than the control group. On the other hand, the BW's of other treated groups were significantly higher than the control group except (DXR+PS/LNE) group was comparable to the control group.
The changes due to different treatments on the heart/ BW ratio are presented in (Table 4). Animals treated with (DXR-Sol, DXR/LNE, DXR+PS/LNE, PS/LNE and DXR+PS-Sol) did not show any significant difference from the control group. However, EAC-challenged, BL-NE and DXR/LNE groups presented a significant drop in the heart/BW ratio. In contrast, the effect of the drug formulations on the liver to the body weight ratio (Table 4) showed no significant change in all groups in comparison to the control group except DXR+PS-Sol group exhibited a significant rise in the liver/BW ratio.

\subsubsection{Assessment of Cardiac Enzymes and Histopathology}

The effect of different treatments on the cardiac enzymes namely CK-MB and LDH activities is presented in (Figures $2 \mathrm{~A} \& 2 \mathrm{~B})$. The observed CK-MB activity has no significant difference for both DXR+PS/LNE and the control groups, but it was significantly raised above that of control group upon treatment with other groups including DXR-Sol, DXR+PS-Sol PS/LNE and DXR/LNE. Although CK-MB activity in all treated groups was within the normal range (50-680 I.U./l) for mice, it was near the upper reference limit in both DXR-Sol and DXR+PS-Sol. Regarding $\mathrm{LDH}$, its activity was significantly elevated in all treated groups when compared to the normal group (Figure 2B). However, the increase in the LDH activity was within the normal range (85- 750 I.U./l) in all treatments, except three groups namely DXR-Sol, DXR+PS-Sol and PS/

Table 3. Effect of different treatments on body weight changes in EAC-bearing mice

\begin{tabular}{|c|c|c|c|}
\hline Animal Group & \multicolumn{3}{|c|}{ Body Weight (g) } \\
\hline & Weight in Day 0 & Weight in Day 15 & Change in weight (g) \\
\hline Control & $28.35 \pm 1.84$ & $30.27 \pm 2.57$ & $1.91 \pm 146 \#$ \\
\hline EAC-challenged & $27.66 \pm 1.75$ & $42.05 \pm 2.04$ & $14.38 \pm 2.42^{\star} \#$ \\
\hline BL-NE & $27.71 \pm 1.74$ & $45.72 \pm 5.68$ & $18.01 \pm 554^{\star} \#$ \\
\hline DXR/LNE & $26.26 \pm 1.02$ & $34.06 \pm 1.73$ & $7.81 \pm 1.61^{\star} \#$ \\
\hline DXR-Sol & $26.91 \pm 1.99$ & $21.09 \pm 2.27$ & $-5.83 \pm 2.54^{\star}$ \\
\hline DXR+PS/LNE & $25.79 \pm 1.84$ & $29.73 \pm 2.13$ & $3.94 \pm 2.59^{\star} \#$ \\
\hline DXR+PS-Sol & $27.08 \pm 1.87$ & $23.68 \pm 4.16$ & $-3.39 \pm 3.33^{\star}$ \\
\hline PS/LNE & $27.09 \pm 1.95$ & $37.21 \pm 5.05$ & $10.17 \pm 5.13^{\star} \#$ \\
\hline
\end{tabular}

Results were expressed as mean $\pm \mathrm{SD}, \mathrm{n}=10$

Statistical analysis was based on one-way ANOVA, and then Tukey's post-hoc test

*Statistically significant differences from the control at $\mathrm{p}<0.05$

"Statistically significant differences from the DXR-Sol group at $\mathrm{p}<0.05$ 
Table 4. Effect of different treatments on heart/body weight and liver/body weight ratios in EAC-bearing mice

\begin{tabular}{|c|c|c|}
\hline Animal Group & Heart/BW & Liver/BW \\
\hline Control & $0.005 \pm 0.001$ & $0.048 \pm 0.007$ \\
\hline EAC-challenged & $0.003 \pm 0.001 \#$ & $0.043 \pm 0.004$ \\
\hline BL-NE & $0.004 \pm 0.001 \#$ & $0.042 \pm 0.003$ \\
\hline DXR/LNE & $0.004 \pm 0.001 \#$ & $0.051 \pm 0.004$ \\
\hline DXR-Sol & $0.006 \pm 0.001$ & $0.048 \pm 0.009$ \\
\hline DXR+PS/LNE & $0.005 \pm 0.001$ & $0.051 \pm 0.007$ \\
\hline DXR+PS-Sol & $0.005 \pm 0.001$ & $0.070 \pm 0.022 \#$ \\
\hline PS/LNE & $0.005 \pm 0.001$ & $0.039 \pm 0.006$ \\
\hline
\end{tabular}

Data were expressed as mean $\pm \mathrm{SD}, \mathrm{n}=10$

Statistical analysis was based on one-way ANOVA, and then Tukey's post-hoc test.

"Statistically significant differences from the corresponding control at $\mathrm{p}<0.05$.

LNE groups. In fact, the rise in LDH activity recorded for the DXR-Sol was higher than that of DXR+PS-Sol group.

Histopathological examination of the mice heart tissues in both the control and EAC-challenged groups showed a normal pattern with no inflammatory cell infiltration (Figures 3A \& 3B). BL-NE treated mice (Figure 3C) showed normal morphology of myocardium roughly similar to the EAC group except endured karyolysis found in some nuclei, while other cells were pyknotic. Treatment with DXR-Sol and DXR+PS-Sol (Figures 3D \& 3E; respectively) showed cardiac tissue damage as manifested by myofibrillar fragmentation, blocking of blood vessels, enhanced vacuolization of cytoplasm with some nuclear changes like karyolysis and pyknosis. Treatment with DXR/LNE, DXR+PS/LNE (Figures 3F \& $3 \mathrm{G}$, respectively) ameliorated myocardial degeneration illustrated by mild disruption of myocardial fibers and decreased vacuolization. As seen in Figure (3H), PS-LNE group developed myocardial degeneration, comprising of blood vessel congestion, myofibril fragmentation, and few nuclei showing karyolytic and pyknotic changes.
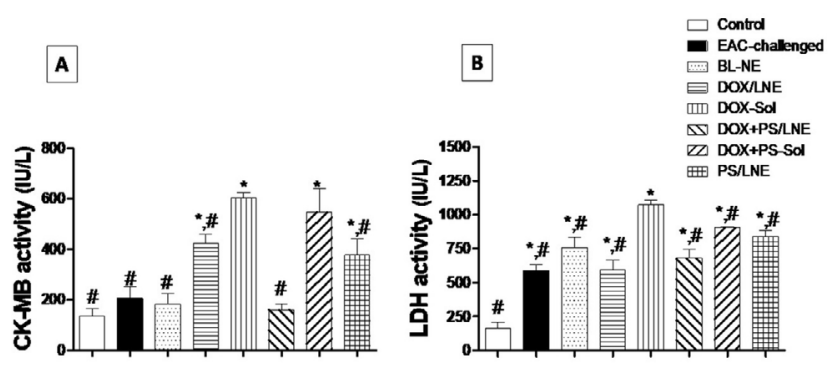

Figure 2. Effect of different drug formulations on A) CK$\mathrm{MB}$ activity, B) LDH activity in EAC bearing mice.
Results were expressed as mean $\pm S D, n=10$. Statistical analysis was based on one-way ANOVA, and then Tukey's post-hoc test. ${ }^{*}$ Statistically significant differences from the corresponding control at $\mathrm{p}<0.05$. "Statistically significant differences from the corresponding DXR-Sol group at $\mathrm{p}$ $<0.05$.

\subsubsection{Assessment of Hepatic Parameters and Histopathology}

The assessed activities include liver enzymes (ALT, AST, ALP) and total bilirubin concentration in EAC bearing mice are shown in Figures 4A, 4B, 4C \& 4D; respectively. All DXR-treated groups including DXR-Sol, DXR/LNE, DXR+PS-Sol and DXR+PS/LNE showed significant increase of all assessed parameters when compared to the corresponding control group. However, DXR+PS/ LNE group was able to ameliorate the harmful effect of DXR treatment, being capable of reducing all measured markers significantly from the DXR-Sol group. In addition, the PS/LNE group showed a significant decline from the DXR-Sol group in all assessed parameters. For ALT and ALP activities (Figure 4A \& 4C), there was a nonsignificant change in EAC-challenged and BL-NE groups, compared to the corresponding control group. However, both groups did have significant rises from the control group for AST activity and total bilirubin concentration (Figures 4B \& 4D).

Light microscopic examination of the hepatic sections of the control and EAC-challenged groups revealed a normal hepatic histological pattern (Figure 5A \& 5B). BL-NE group showed some vacuoles formation (Figure 5C). The hepatocytes were arranged as sheets close 

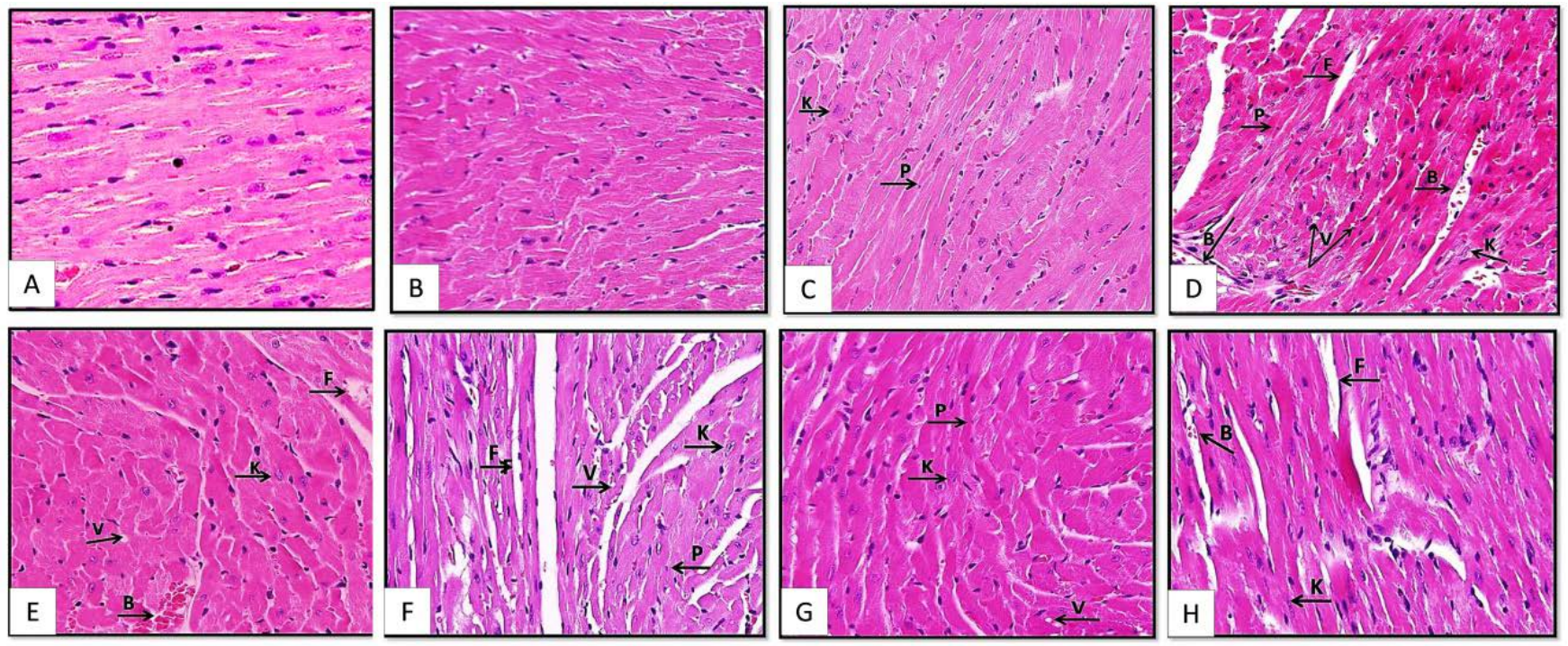

Figure 3. Histopathological examination of mice cardiac muscle stained by H\&E (X400).

A) Control group shows normal cardiac muscle architecture; B) EAC-challenged group shows normal cardiac muscle architecture; C) BL-NE group shows few cardiac muscles enduring karyolysis (K) while others are pyknotic (P); D) DXR-Sol group shows damaged cardiomyocytes manifested by enhanced vacuolization (V), myofibrillar fragmentation (F) and congestion of blood vessel (B); E) DXR/LNE group reveals minor morphological alteration of the cardiac muscles with mild degree of vacuolization and damage; F) DXR+PS-Sol group shows marked fragmentation (F), vacuolization (V) and some karyolytic (K) and pyknotic (P) nuclei; G) DXR+PS/LNE group shows almost normal cardiac muscle architecture with some changes like karyolysis (K), pyknosis $(\mathrm{P})$, and cytoplasmic vacuolization (V); H) PS/LNE group shows blood vessels congestion (B), fragmentation of myofibrils (F) and few karyolytic nuclei (K).

to the central vein in an unsophisticated and regular way in a normal hepatic architecture. In contrast, mice received (DXR-Sol) treatment showed massive pathological alterations (Figure 5D), manifested as marked deterioration of hepatic tissue, appeared as empty vacuoles. Many hepatic cells attached together, forming areas of degenerated cells and losing normal histological characteristics. Moreover, the central vein and sinusoidal spaces were dilated and congested, accompanied by activation of the inflammatory Kupffer cells. Interestingly, the (DXR+PS-Sol) group underwent milder pathological alterations as compared to (DXR-Sol) group as detected in (Figure 5F), evident by modest degree of degenerated hepatocytes, central vein dilation, proliferation of activated Kupffer cells and cytoplasmic vacuolization. On the contrary, hepatic histological findings of (DXR/ $\mathrm{LNE})$, and (DXR+PS/LNE) groups-shown in (Figures 5E \& 5G; respectively) - were comparable to the control group, showing almost normal hepatic architecture. The (PS/LNE) group showed degeneration of few hepatocytes, moderate hemorrhage in central vein, and presence of Kupffer cells (Figure 5H).
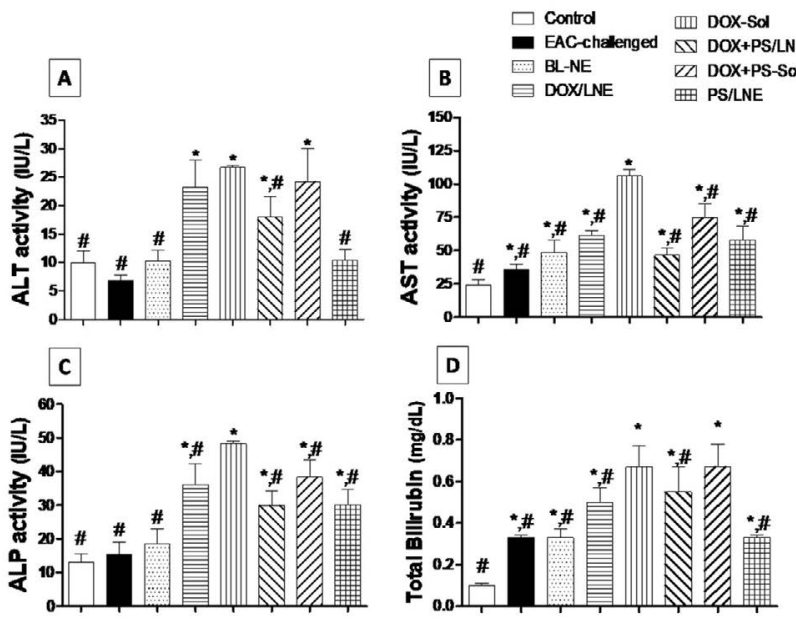

Figure 4. Effect of different drug formulations on serum enzymatic activities of A) ALT, B) AST, C) ALP in addition to D) serum total bilirubin concentration in EAC bearing mice. 
Table 5. The effect of different treatments on MST, \% ILS and \% S

\begin{tabular}{|c|c|c|c|}
\hline Animal Group & MST (days) & \%ILS & $\%$ S \\
\hline Control & $80.0 \pm 2.56$ & - & 100 \\
\hline EAC-challenged & $21.9 \pm 1.73$ & - & 0 \\
\hline BL-NE & $22.5 \pm 2.22$ & $265.29^{*}$ & $100^{*}$ \\
\hline DXR/LNE & $80.0 \pm 3.25$ & $57.99^{*}$ & 0 \\
\hline DXR-Sol & $34.6 \pm 8.88$ & $217.35^{*}$ & $70^{*}$ \\
\hline DXR+PS/LNE & $69.5 \pm 17.62$ & $122.83^{*}$ & $30^{*}$ \\
\hline DXR+PS-Sol. & $48.8 \pm 25.06$ & 9.59 & 0 \\
\hline PS/LNE & $24.0 \pm 2.94$ & & \\
\hline
\end{tabular}

Results were expressed as mean $\pm \mathrm{SD}, \mathrm{n}=10$

Statistical analysis was based on one-way ANOVA, and then Tukey's post-hoc test

*Statistically significant differences from the corresponding EAC-challenged group at $\mathrm{p}<0.05$

Results were expressed as mean $\pm S D, n=10$. Statistical analysis was based on one-way ANOVA, and then Tukey's post-hoc test. *Statistically significant differences from the corresponding control at $\mathrm{p}<0.05$. ${ }^{\text {"Statistically significant }}$ differences from the corresponding DXR-Sol group at $\mathrm{p}$ $<0.05$.
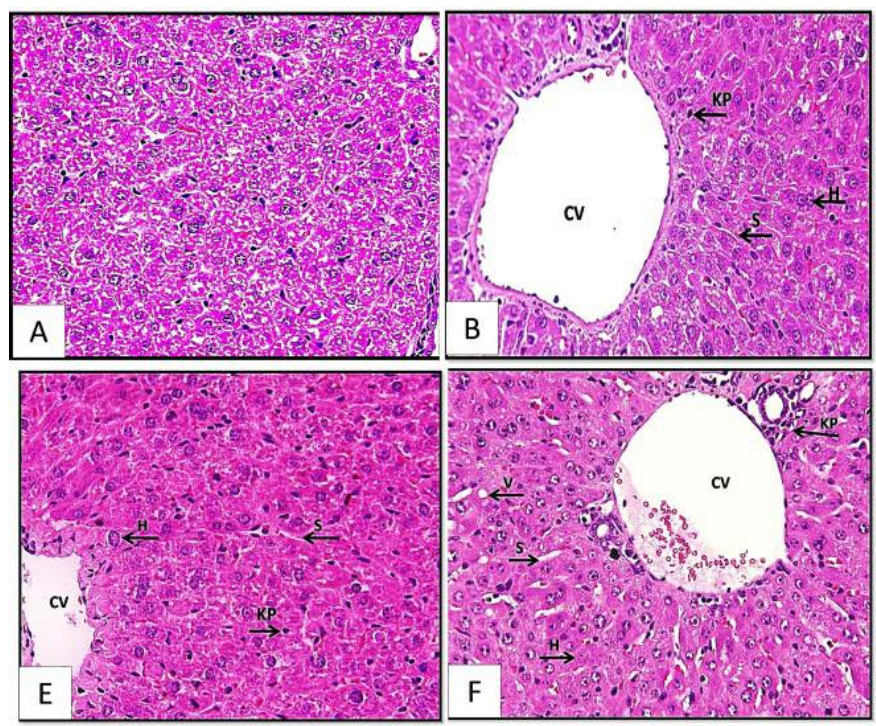

\subsubsection{Survival Study}

The effect of tested formulations on mean survival time (MST), percentage increase in lifespan (\% ILS) and survival percentage (\% S) is illustrated in (Table 5). The MST of the EAC-challenged group did not significantly

Figure 5. Histopathological examination of mice liver stained by $H \& E(X 400)$.

A) Control group exhibits normal histopathological structure; B) EAC-challenged group is similar to the previous control group; C) BL-NE group shows radially-arranged hepatocytes with some vacuoles formation (H) surrounding the central vein (CV) and blood sinusoids (S) with vacuoles (V) and Kupffer cells (KP); D) DXRSol group reveals pronounced degenerated hepatic cells $(\mathrm{H})$ with the central vein $(\mathrm{CV})$ markedly dilated with hemorrhage and the blood sinusoids (S) are infiltrated with RBCs (RC) and activated Kupffer cells (KP); E) DXR/ LNE group presents nearly normal histological structure of the liver; F) DXR+PS-Sol group exhibits moderate degenerated hepatocytes $(\mathrm{H})$, mild degree of central vein dilation $(\mathrm{CV})$, activated Kupffer cells (KP) and vacuoles (V); G) DXR + PS/LNE group shows almost normal hepatic architecture; H) PS/LNE group displays moderate degree of central vein hemorrhage (CV), few degenerated hepatocytes (H) with some Kupffer cells (KP). 
vary from those of (PS/LNE) and (BL-NE) groups. Yet, a significant difference was observed in the MST of the EAC-challenged group and all the treated groups receiving DXR. Interestingly, the highest MST $(80 \pm 0$ days) was recorded for the DXR/LNE group, whereas the lowest MST (34.6 \pm 8.877 days) was recorded in the DXRSol group. The MST of (DXR+PS/LNE) was significantly greater than that of (DXR+PS-Sol). The increases in lifespan (\% ILS) in (DXR/LNE), (DXR+PS/LNE) and (DXR+PS-Sol) were 4.5, 3.75 and 2.11 folds, respectively; as compared to (DXR-Sol) group. It is worthy noted that the $\%$ ILS of (DXR+PS/LNE) was 1.8-fold greater than that of (DXR+PS-Sol). Further, the survival percentage (\% S) of (DXR+PS/LNE) and (DXR+PS-Sol) were $70 \%$ and $30 \%$ respectively, while the (DXR/LNE) group had a $100 \%$ survival percentage.

\section{Discussion}

Cancer is one of the major causes of mortality worldwide and the failure of conventional chemotherapy to reduce mortality rates indicates that there is a critical requirement of new approaches ${ }^{1}$. Many anticancer drugs are non-specific, thus they accumulate both in tumorous cells and the normal cells. It is well established that newer approaches in drug delivery are more specific thus limits the toxicity of anticancer agents. Few experimental studies have shown that the antitumor agents bound to polymeric nanoparticle prolongs drug retention in tumors, increase survival of tumor bearing animals and shrinks tumor growth $^{28,29}$. Experimental and epidemiological studies have suggested that statins (3 HMG-CoA reductase inhibitors) may have potential protective effect against cancer progression. A few experimental studies suggested that statins can induce synergistic antitumor activity when combined with conventional chemotherapeutics ${ }^{14}$.

LNE size is an important factor determining the rate of activity, cellular interaction, internalization and subcellular distribution ${ }^{30}$. In our study, the mean droplet sizes of DXR/LNE were similar to other previous studies demonstrating incorporation of DXR into the NE formulations ${ }^{24,25}$. The mean droplet diameter of DXR+PS/ LNE formulation was more than 16 and 6-fold larger than the droplet sizes of the DXR/LNE and PS/LNE suggesting successful encapsulation of DXR and PS. The amount of hydrophilic PS was twice the amount of DXR which might enhance the steric hindrance between the drugs and could reduce the droplets packing.

Animals body weight is considered as an indicator of general health in models of chemotherapy-induced fatigue. In confirmation with earlier studies, there was a significant more than 7-fold weight gain in EAC challenged group ${ }^{27}$. Compared to the EAC challenged, DXR+PS/LNE treated animals showed a significant more than 3 -fold weight loss. However, animals treated with DXR-Sol showed around 2 -fold weight loss than DXR+PS/LNE indicating reduced tumor burden. Our results were similar to prior studies which reported that DXR causes significant weight loss of rats $^{31}$. The weight loss in DXR group could be attributed to the diminished appetite that might be due to peripheral (stomatitis and gastroenteritis) and/or central (induction of chemotherapy trigger zone or vomiting center) mechanisms ${ }^{32}$. Moreover, we observed that the DXR/LNE group had a significant weight loss as compared to the EAC challenged group. A previous study reported that PS inhibited cellular proliferation of hepatocarcinoma cells both in in-vitro and in-vivo models ${ }^{33}$. On comparing the results of our study with earlier studies, treatment with the DXR+PS/LNE restricted both weight loss and weight gain as observed with DXR and EAC challenged groups, respectively.

In the present study we assessed the ratio of organ weight to body weight to evaluate the toxicity of the drug formulations on the organ. No significant change was observed in ratio of heart weight to body weight in mice treated with DXR $2 \mathrm{mg} / \mathrm{kg}$ in comparison to the normal group. However, a significant increase in liver weight to body weight ratio of DXR+PS-Sol treated group was observed as compared to the normal group. Unlike DXR+PS/LNE, DXR/LNE and DXR treated groups did not show any change in liver weight to body weight ratio. It was reported that rats treated with DXR $15 \mathrm{mg} / \mathrm{kg}$ for 2 weeks have considerable increase in ratios of heart and liver weights to body weight as compared to untreated rats $^{15}$. An enlarged liver (hepatomegaly) is a symptom of an underlying disease, such as hepatic disorder, heart failure or cancer. Inflammation caused due to infection, toxins, drugs and an increased Kupffer cell activation are some of the mechanisms related to hepatomegaly ${ }^{34}$. From our results, we found that DXR+PS/LNE offers better protection for hepatomegaly as compared to the DXR+PS-Sol. 
DXR is a very potent antibiotic with antitumor activity but its use is limited because of its toxic effects on heart and liver as it has been established in various animal models ${ }^{31}$. In a malignant tumor, the tumor marker enzyme change reflects an overall metabolic change and thereby, serves as crucial parameter for tumor cell sensitivity and specificity. Biochemical parameters and enzymatic activities of NE formulations were monitored to evaluate the cardiotoxicity and hepatotoxicity. The findings of our study revealed that mice administered with DXR-Sol and DXR+PS-Sol had a significant raise in the serum hepatic enzymes levels (ALT, AST and ALP) compared to the normal group. The elevated activities of serum transaminases (AST and ALT) are related to hepatic dysfunction in tumor-bearing mice. These findings are in agreement with an earlier study which reported a marked increase in serum ALT, AST, and ALP activities manifested as hepatotoxicity post DXR treatment intraperitoneally at a cumulative dose of $12 \mathrm{mg} / \mathrm{kg}^{35}$. ALP is an effective anti-inflammatory mediator; it protects tissues from damage due to injury. Thus, an elevated ALP in the DXR group may account for prominent tissue injury and inflammation.

The observed histopathological changes in the liver of DXR-Sol treated mice was degenerated hepatocytes, marked congestion of central vein with hemorrhage, dilated sinusoidal spaces infiltrated with red blood cells, and activated Kupffer cells. These changes of DXR-Sol group were comparable to earlier reports ${ }^{36}$. Also, in DXR-Sol and DXR+PS-Sol groups, there were significant elevation of cardiac injury markers (LDH and $\mathrm{CK}-\mathrm{MB}$ ), which corroborates with clinical findings during follow-up of DXR therapy ${ }^{37}$. In agreement with our findings, a previous study reported that a single dose of DXR in rats showed elevations in cardiac enzymes levels due to generation of free radicals and increased permeability of myocardial membrane ${ }^{38}$. This was further substantiated by the light microscopy images of cardiac tissue sections of mice treated with the DXR-Sol, which exhibited severe pathological alterations, while normal architecture was noticed in the EAC-challenged mice. DXR-Sol induced myocardial changes were characterized by the myofibrillar loss, congestion of myocardial vessels, cytoplasmic vacuolization and perinuclear changes like karyolysis and pyknosis.

The DXR+PS/LNE treated animals had significant declined levels of ALT, AST, CK-MB and LDH in comparison with DXR-Sol group; in contrast these enzymatic levels were not significantly different from control group indicating strong protective activity against DXR toxicity. It was confirmed that nanoparticles loaded with DXR offers cardio-protection reported as decrease in CPK level compared to the DXR administered group $^{39}$. Multiple studies demonstrated that DXRinduced myocardial toxicity and hepatic injury can be protected by statins due to potential antioxidative and anti-inflammatory effects ${ }^{8,9,40}$. Our study confirmed these findings and suggests that the presence of nanoemulsion formulation of DXR+PS may offer protection to the myocardial and hepatic tissue against DXR.

In contrast to DXR-Sol group, the cardiac and hepatic tissues of DXR+PS/LNE and EAC challenged group sections were having normal tissues architecture. These findings indicate that combination of PS with DXR as loaded NE formulation reduced DXR induced hepatocardiac toxicity. We observed that all drug formulations had a significant increase in BIL levels as compared to the control group. An elevated serum total BIL may be caused by blocking of bile ducts leading to regurgitation of conjugated BIL from the damaged hepatocytes and inflammation ${ }^{35}$.

Life-span prolongation in a tumor-bearing animal is considered a reliable criterion for estimating the worth of any chemotherapeutic agent ${ }^{41}$. DXR-Sol treated EAC bearing mice had significantly less life span parameters (MST, \% ILS and \% S) than DXR/LNE treated EAC bearing mice. Also, NE formulation of DXR + PS (DXR+PS/LNE) significantly prolonged MST, \% ILS and $\% \mathrm{~S}$ of the EAC bearing mice compared to the DXR+PS combination (DXR+PS-Sol) and DXR. Our findings are in agreement with another previous finding which revealed that the nanoparticles also decrease DXR related toxicity thereby resulting in a significant prolongation in average life-span as compared to free DXR ${ }^{42}$. Similarly, multiple reports have shown that PS improved patient survival in advanced hepato-carcinoma ${ }^{33,43}$.

\section{Conclusions}

We found that the combination of DXR with PS loaded in a NE has significantly enhanced tolerability of DXR against the EAC bearing mice. These facts open a new avenue for combination therapy to be used in cancer. However, 
extensive tolerability and efficacy studies in animals and humans are required to confirm these observations.

\section{Acknowledgements}

This project was funded by the Deanship of Scientific Research (DSR) at King Abdulaziz University, Jeddah (grant no. 189/247/1433). The authors, acknowledge with thanks the DSR for technical and financial support.

\section{Conflict of Interest}

The authors declare that they have no competing interests.

\section{References}

1. Lane RJ, Khin NY, Pavlakis N, Hugh TJ, Clarke SJ, Magnussen J, Rogan C, Flekser RL. Challenges in chemotherapy delivery: comparison of standard chemotherapy delivery to locoregional vascular mass fluid transfer. Future Oncol. 2018; 14(7): 647-663. https://doi.org/10.2217/fon-20170546.

2. Tocchetti CG, Cadeddu C, Di Lisi D, Femminò S, Madonna R, Mele D, Monte I, Novo G, Penna C, Pepe A, Spallarossa P, Varricchi G, Zito C, Pagliaro P, Mercuro G. From molecular mechanisms to clinical management of antineoplastic drug-induced cardiovascular toxicity: A translational overview. Antioxid Redox Signal. 2017; 30(18):2110-2153. https://doi.org/10.1089/ars.2016.6930

3. Cai F, Luis M, Lin X, Wang M, Cai L, Cen C, Biskup E. Anthracycline-induced cardiotoxicity in the chemotherapy treatment of breast cancer: Preventive strategies and treatment. Mol Clin Oncol. 2019; 11(1): 15-23. https://doi. org/10.3892/mco.2019.1854.

4. Alkreathy H, Damanhouri ZA, Ahmed N, Slevin M, Ali SS, Osman AMM. Aged garlic extract protects against doxorubicin-induced cardiotoxicity in rats. Food Chem Toxicol. 2010; 48(3): 951-956. https://doi.org/10.1016/j. fct.2010.01.005.

5. Sadeghi-Aliabadi H, Minaiyan M, Dabestan A. Cytotoxic evaluation of doxorubicin in combination with simvastatin against human cancer cells. Res Pharm Sci. 2010; 5(2): 127-133.

6. Zheng J, Lee HCM, bin Sattar MM, Huang Y, Bian JS. Cardioprotective effects of epigallocatechin-3-gallate against doxorubicin-induced cardiomyocyte injury. Eur J Pharmacol. 2011; 652(1-3): 82-88. https://doi. org/10.1016/j.ejphar.2010.10.082.

7. Cagel M, Grotz E, Bernabeu E, Moretton MA, Chiappetta DA. Doxorubicin: nanotechnological overviews from bench to bedside. Drug Discov Today. 2017; 22(2): 270281. https://doi.org/10.1016/j.drudis.2016.11.005.

8. Alkreathy HM, Alkhatib MH, Al Musaddi SA, Balamash KSA, Osman NN, Ahmad A. Enhanced anti-tumor activity of doxorubicin and simvastatin combination loaded nanoemulsion treatment against a Swiss albino mouse model of Ehrlich ascites carcinoma. Clin Exp Pharmacol Physiol. 2019; 46(5): 496-505. https://doi. org/10.1111/1440-1681.13071.

9. Alkreathy HM, Alkhatib MH, Al-thepyani MAH, Balamash KSA, Alghamdi SK, Karim S, Ahmad A. Synergistic antitumor activity of doxorubicin and atorvastatin combination loaded nanoemulsion in mice. Int $\mathrm{J}$ Pharmacol. 2020; 16(4): 330-342. https://doi.org/10.3923/ ijp.2020.330.342.

10. Liu S, Li R, Qian J, Sun J, Li J, Shen J, Xie Y. Combination Therapy of Doxorubicin and Quercetin on MultidrugResistant Breast Cancer and Their Sequential Delivery by Reduction-Sensitive Hyaluronic Acid-Based Conjugate/da-Tocopheryl Poly(ethylene glycol) 1000 Succinate Mixed Micelles. Mol Pharm. 2020; 17(4):1415-1427. https://doi. org/10.1021/acs.molpharmaceut.0c00138.

11. Stroes E. Statins and LDL-cholesterol lowering: an overview. Curr Med Res Opin. 2005; 21 Suppl 6: S9-S16. https://doi. org/10.1185/030079905X59102.

12. Ramkumar S, Raghunath A, Raghunath S. Statin Therapy: Review of Safety and Potential Side Effects. Acta Cardiol Sin. 2016; 32(6): 631-639. https://doi.org/10.6515/ ACS20160611A.

13. Bil J, Zapala L, Nowis D, Jakobisiak M, Golab J. Statins potentiate cytostatic/cytotoxic activity of sorafenib but not sunitinib against tumor cell lines in vitro. Cancer Lett. 2010; 288(1): 57-67. https://doi.org/10.1016/j. canlet.2009.06.022.

14. Zaleska M, Mozenska O, Bil J. Statins use and cancer: an update. Future Oncol. 2018; 14(15): 1497-1509. https://doi. org/10.2217/fon-2017-0543.

15. Koti BC, Nagathan S, Vishwanathswamy A, Gadad PC, Thippeswamy A. Cardioprotective effect of Vedic Guard against doxorubicin-induced cardiotoxicity in rats: A biochemical, electrocardiographic, and histopathological study. Pharmacogn Mag. 2013; 9(34): 176-181. https://doi. org/10.4103/0973-1296.111287.

16. Henslee A B, Steele TA. Combination statin and chemotherapy inhibits proliferation and cytotoxicity of an aggressive natural killer cell leukemia. Biomark Res. 2018; 6(1): 26. https://doi.org/10.4103/0973-1296.111287.

17. Martirosyan A, Clendening JW, Goard CA, Penn LZ. Lovastatin induces apoptosis of ovarian cancer cells and synergizes with doxorubicin: potential therapeutic relevance. BMC Cancer. 2010; 10(1): 103. https://doi. org/10.1186/1471-2407-10-103. 
18. Wang A, Wakelee HA, Aragaki AK, Tang JY, Kurian AW, Manson JE, Stefanick ML. Protective effects of statins in cancer: should they be prescribed for high-risk patients? Curr Atheroscler Rep. 2016; 18(12):72. https://doi. org/10.1007/s11883-016-0625-y.

19. Cholesterol Treatment Trialists' (CTT) Collaborators. Efficacy and safety of LDL-lowering therapy among men and women: meta-analysis of individual data from 174 000 participants in 27 randomised trials. Lancet. 2015; 385 (9976): 1397-1405. https://doi.org/10.1016/S01406736(14)61368-4.

20. Simon MS, Rosenberg CA, Rodabough RJ, Greenland P, Ockene I, Roy HK, Lane DS, Cauley JA, Khandekar J. Prospective analysis of association between use of statins or other lipid-lowering agents and colorectal cancer risk. Ann Epidemiol. 2012; 22(1):17-27. https://doi.org/10.1016/j. annepidem.2011.10.006.

21. Murtola TJ, Visvanathan K, Artama M, Vainio H, Pukkala E. Statin use and breast cancer survival: a nationwide cohort study from Finland. PloS One. 2014; 9(10): e110231. https://doi.org/10.1371/journal.pone.0110231.

22. Cardwell CR, Hicks BM, Hughes C, Murray LJ. Statin use after diagnosis of breast cancer and survival: a populationbased cohort study. Epidemiology. 2015; 26(1): 68-78. https://doi.org/10.1097/EDE.0000000000000189.

23. Sánchez-López E, Guerra M, Dias-Ferreira J, LopezMachado A, Ettcheto M, Cano A, Espina M, Camins A, Garcia M L, Souto EB. Current Applications of Nanoemulsions in Cancer Therapeutics. Nanomaterials (Basel, Switzerland). 2019; 9(6): 821. https://doi. org/10.3390/nano9060821.

24. Alkhatib MH, Albishi HM, Mahassni SH. Impact of nanoparticles on cancer therapy. Trop J Pharm Res. 2012; 11(6):1001-1011. https://doi.org/10.4314/tjpr.v11i6.18.

25. Alkhatib $\mathrm{MH}, \mathrm{AlBishi} \mathrm{HM}$. In vitro evaluation of antitumor activity of doxorubicin-loaded nanoemulsion in MCF-7 human breast cancer cells. J Nanopart Res. 2013; 15(3): 1489. https://doi.org/10.1007/s11051-013-1489-5.

26. Alkreathy HM, Damanhouri ZA, Ahmed N, Slevin M, Osman AMM. Mechanisms of cardioprotective effect of aged garlic extract against doxorubicin-induced cardiotoxicity. Integr Cancer Ther. 2012; 11(4): 364-370. https://doi.org/10.1177/1534735411426726.

27. Raju AB, Ravindranath A, Kalpana G. Antitumor activity of Diospyrosperegrina on Ehrlich ascites carcinoma in mice. J Sci Res. 2011; 3(2): 413-419. https://doi.org/10.3329/jsr. v3i2.6787.

28. Massing U, Fuxius S. Liposomal formulations of anticancer drugs: selectivity and effectiveness. Drug Resist Updat. 2000; 3(3):171-177. https://doi.org/10.1054/drup.2000.0138.

29. Karlsson J, Vaughan HJ, Green JJ. Biodegradable Polymeric Nanoparticles for Therapeutic Cancer Treatments. Annu
Rev Chem Biomol Eng. 2018; 9: 105-127. https://doi. org/10.1146/annurev-chembioeng-060817-084055.

30. Danaei M, Dehghankhold M, Ataei S, Hasanzadeh Davarani F, Javanmard R, Dokhani A, Khorasani S, Mozafari MR. Impact of particle size and polydispersity index on the clinical applications of lipidic nanocarrier systems. Pharmaceutics. 2018; 10(2): 57. https://doi.org/10.3390/ pharmaceutics 10020057.

31. Rašković A, Stilinović N, Kolarović J, Vasović V, Vukmirović S, Mikov M. The protective effects of silymarin against doxorubicin-induced cardiotoxicity and hepatotoxicity in rats. Molecules. 2011; 16(10):8601-8613. https://doi. org/10.3390/molecules16108601.

32. Zombeck JA, Fey EG, Lyng GD, Sonis ST. A clinically translatable mouse model for chemotherapy-related fatigue. Comp Med. 2013; 63(6): 491-497.

33. Hijona E, Banales JM, Hijona L, Medina JF, Arenas J, Herreros-Villanueva M, Aldazabal P, Bujanda L. Pravastatin inhibits cell proliferation and increased MAT1A expression in hepatocarcinoma cells and in vivo models. Cancer Cell Int. 2012; 12(1): 5. https://doi.org/10.1186/1475-2867-125.

34. Kubitz R, Merker G, Häussinger D. Hepatomegaly. Encyclopedia of Molecular Mechanisms of Disease. 2009; 824-825.

35. Soliman HA, Ahmed RR, Ali AT. Assessment of the chemopreventive effects of various plant constituents against doxorubicin-induced toxicity in rats. J Am Sci. 2014; 10:153-164.

36. Salouege I, Ben Ali R, Ben Saïd D, Elkadri N, Kourda N, Lakhal M, Klouz A. Means of evaluation and protection from doxorubicin-induced cardiotoxicity and hepatotoxicity in rats. J Cancer Res Ther. 2014; 10(2): 274-278. https://doi. org/10.4103/0973-1482.136557.

37. Divakaran SA, Nai CK. Amelioration of doxorubicin induced cardiotoxicity in tumor bearing mice by ferulic acid: a mechanistic study at cellular and biochemical level. Int J Tumor Therap. 2012; 1 (2): 6-13. https://doi. org/10.5923/j.ijtt.20120102.01.

38. Gandhi H, Patel V, Mistry N, Patni N, Nandania J, Balaraman R. Doxorubicin mediated cardiotoxicity in rats: Protective role of felodipine on cardiac indices. Environ Toxicol Pharmacol. 2013; 36: 787-795. https://doi.org/10.1016/j. etap.2013.07.007.

39. Park J, Fong PM, Lu J, Russell KS, Booth CJ, Saltzman WM, Fahmy TM. PEGylated PLGA nanoparticles for the improved delivery of doxorubicin. Nanomedicine. 2009; 5(4):410-418. https://doi.org/10.1016/j.nano.2009.02.002.

40. Henninger C, Fritz G. Statins in anthracycline-induced cardiotoxicity: Rac and Rho, and the heartbreakers. Cell Death Dis. 2017; 8(1): e2564-e2564. https://doi. org/10.1038/cddis.2016.418. 
41. Haldar PK, Kar B, Bala A, Bhattacharya S, Mazumder UK. Antitumor activity of Sansevieria roxburghiana rhizome against Ehrlich ascites carcinoma in mice. Pharm Biol. 2010; 48(12): 1337-1343. https://doi. org/10.3109/13880201003792592.

42. Hao H, Ma Q, Huang C, He F, Yao P. Preparation, characterization, and in vivo evaluation of doxorubicin loaded BSA nanoparticles with folic acid modified dextran surface. Int J Pharm. 2013; 444(1-2): 77-84. https://doi. org/10.1016/j.ijpharm.2013.01.041.

43. Kawata S, Yamasaki E, Nagase T, Inui $Y$, Ito N, Matsuda Y, Inada M, Tamura S, Noda S, Imai Y, Matsuzawa Y. Effect of pravastatin on survival in patients with advanced hepatocellular carcinoma. A randomized controlled trial. Br J Cancer. 2001; 84(7): 886-891. https://doi.org/10.1054/ bjoc. 2000.1716 . 